\title{
Gestores de contenido en la arquitectura de la producción multiplataforma. El caso de Televisió de Catalunya
}

\author{
Por Rosa Franquet y María-Isabel Villa-Montoya
}

\begin{abstract}
Resumen: Se analiza un gestor de contendidos adaptado a las necesidades de Televisión de Catalunya a partir de un software que se comercializó con el nombre de DeliverTy, como sistema tecnológico para la producción audiovisual multiplataforma. Se plantean las características de este software, las ventajas que conlleva su implementación y se describen las implicaciones que se derivan de su uso en las diferentes fases de producción de contenidos multiplataforma. Del análisis se extraen conclusiones sobre las contribuciones de los gestores de contenidos a la producción de la información. Entre las ventajas del sistema se puede destacar la simplificación de las tareas de administración de los documentos y archivos generados por los trabajadores del medio televisivo y la facilidad para derivar dichos contenidos hacia las nuevas plataformas.

Palabras clave: Gestores de contenido, Digitalización, CCMA, Televisió de Catalunya, Multiplataforma, Rutinas productivas, Gestión audiovisual, Internet, Web.

Title: Content management systems on multiplatform production architecture. The case of Televisió de Catalunya

Abstract: This article examines an software adapted in-house from DeliverTy, a content management system, as a technological system for the multiplatform audiovisual production of the autonomous television network of Catalonia. We analyze the features of this software, discuss the benefits associated with its use, and elaborate on the different phases of digital media production. The study draws conclusions on the contribution of content management systems to information production. Among the advantages that stand out are the simplification of the tasks related to document management and files generation, as well as the simplification of the process to broadcast these contents on new platforms.
\end{abstract}

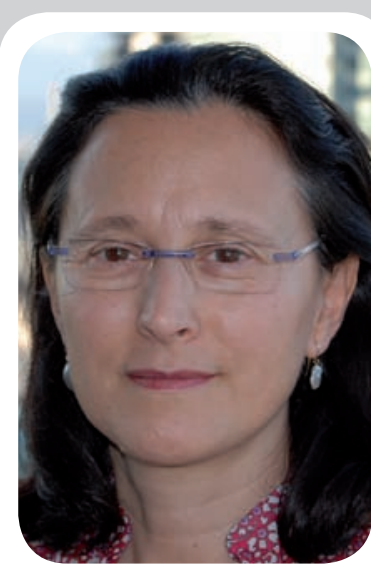

Rosa Franquet es catedrática de comunicación audiovisual y publicidad de la Universitat Autònoma de Barcelona y coordinadora académica de los estudios de doctorado "Contenidos de comunicación en la era digital" y del máster oficial "Investigación en contenidos en la era digital". Es directora de proyectos del Griss (Grupo de Investigación en Imagen, Sonido y Síntesis). Presidenta de la Societat Catalana de Comunicació de l'Institut d'Estudis Catalans. Ha sido investigadora y profesora invitada en universidades nacionales e internacionales. Es especialista en comunicación interactiva, radio y televisión y ha publicado numerosos artículos y libros.

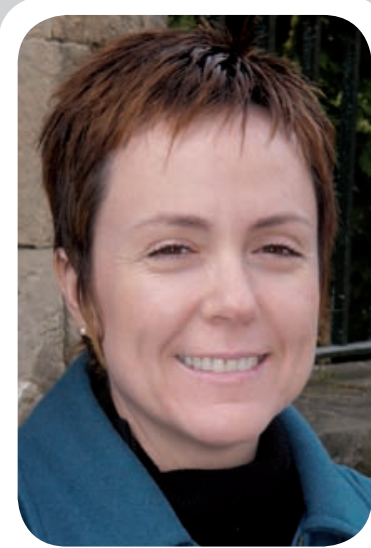

María-Isabel Villa-Montoya es becaria predoctoral de formación del personal investigador de la Universitat Autònoma de Barcelona, integrada Griss (Grupo de Investigación en Imagen, Sonido y Síntesis). Es licenciada en comunicación social y periodismo por la Universidad Pontificia Bolivariana y DEA en comunicación audiovisual por la Universitat de Barcelona, donde lleva a cabo su tesis doctoral sobre los cambios de la televisión en el paso a las nuevas plataformas de emisión. Sus campos de interés son la imagen y las nuevas tecnologías de la comunicación. Hasta ahora ha desempeñado su actividad profesional en diversos medios de comunicación como periodista y fotógrafa.

Keywords: Content management system, Digitization, CCMA, Televisió de Catalunya, Multiplatform, Productive routines, Audiovisual management, Internet, Web.

Franquet, Rosa; Villa-Montoya, María-Isabel. "Gestores de contenido en la arquitectura de la producción multiplataforma. El caso de Televisió de Catalunya”. El profesional de la información, 2010, julio-agosto, v. 19, n. 4, pp. $389-394$.

DOI: $10.3145 /$ epi.2010.jul.08

\section{Introducción}

\section{LA APARIENCIA VISUAL} de los medios online, el diseño y los procedimientos de interacción han recibido una especial atención en los trabajos sobre la arquitectura de la información en los últimos años. En cambio, los equipos, las técnicas y los méto- dos que cada organización emplea en la configuración de los mensajes audiovisuales han sido menos explorados.

Si la arquitectura de edificios incluye las técnicas constructivas, el análisis de la arquitectura de la información debe contemplar los gestores de contenido (en adelante content management systems, CMS) como base de los procesos de indexación, catalogación y emisión. En concreto, la implementación de los CMS en las empresas televisivas altera las rutinas productivas y la oferta de servicios desde las diferentes plataformas de distribución disponibles. El software es más que una simple 
mejora del proceso y constituye una pieza esencial en la arquitectura de la información que se va a suministrar. En la línea de lo que Manovich (2008, p. 5) afirma, "el estudio del software en la sociedad de la información es comparable al estudio de la electricidad y la combustión en la sociedad industrial".

La mayor parte de los productos que proporcionan las cadenas de televisión mediante internet y otros dispositivos capaces de recibir señal digital están mediados por los $C M S$, los cuales han contribuido a la evolución de los contenidos al hacer posible su almacenamiento, su mezcla o su enlace con independencia del formato con el que fueron creados.

\section{"La implementación de los CMS en las empresas televisivas ha mejorado las rutinas productivas y sus servicios"}

Como se verá a lo largo de este artículo, los efectos de los CMS repercuten en la producción multiplataforma, ya que los profesionales trabajan con contenidos en red que pueden compartir, y al mismo tiempo disponen de acceso a fuentes de archivo y sistemas de publicación (Vila, 2009). Ello facilita la creación de servicios transversales que aprovechan las nuevas posibilidades técnicas para aumentar el disfrute de la audiencia.

\section{Aportación de la tecnología}

En la última década el uso de los $C M S$ se ha extendido entre empresas de diversa índole. En los hospitales sirven para gestionar la información médica de los pacientes, en las compañías con fines comerciales para administrar los sitios web corporativos (Serrano-Cobos, 2007) y en las bibliotecas para hacer sus espacios online tan ricos y variados como sus catálogos (Seadle, 2006).

Los CMS constituyen una solución a las necesidades de administración, almacenamiento, publicación y empaquetado de textos, audios, animaciones, gráficos y vídeos. Los documentos digitales, al incorporar los metadatos, pueden recuperarse con facilidad y las búsquedas son precisas y personalizadas.

Estas prestaciones representan para los medios de comunicación un incremento de la productividad, una reducción de los costes, un reaprovechamiento del material de archivo y un notable aumento de la capacidad creativa de los equipos humanos involucrados en la producción (Jeffery-Poulter, 2003).

Los $C M S$ actuales se basan en el xml (extensible markup language), capaz de facilitar la adaptación de piezas a varios formatos. En los sistemas $\mathrm{xml}$, el contenido se almacena dentro de etiquetas del tipo $<\mathrm{a}></ \mathrm{a}>$ y el diseño se aplica con xslt (extensible stylesheet language transformations), un lenguaje de hojas de estilo (Benzing, 2006). Para que los $C M S$ sean útiles en la provisión de servicios multiplataforma deben contener además del propio gestor encargado de organizar, almacenar y emitir, un repositorio central, un sistema de workflow (con documentos que se van procesando por etapas) y una aplicación para administrar las operaciones del software, su configuración y sus usuarios (Schneider, 2008).

Con esta solución técnica, el profesional de la información evita la codificación manual para cada plataforma sin invertir un tiempo extra en la adaptación a cada soporte. No es necesario conocer la disposición, el diseño o el lenguaje de programación de los sitios web, basta con seguir una plantilla preestablecida.
Ante la abundancia de fuentes, formatos y logotipos presentes en la elaboración de los diferentes programas de televisión, los $C M S$ evitan que la organización y la apariencia de las webs de los canales y sus servicios de vídeo online pueda ser caótica o desordenada, ahorran tiempo en la publicación y consiguen una imagen corporativa más homogénea con la aplicación de plantillas diseñadas con una línea gráfica común para todos los sitos web de la empresa.

En síntesis los CMS son utilizados en las cadenas de televisión para administrar las bases de datos digitales, localizar los contenidos y publicar mediante distintas plataformas de forma ágil y automática.

"Con los CMS se administran las bases de datos, se localizan los contenidos y se publica de forma ágil y automática"

\section{Adopción y funcionamiento de DeliverTy}

La televisión atraviesa un estado de mutación y redefinición que afecta a su relación con los espectadores y su posición en el conjunto de sistemas de comunicación. Con la digitalización de la señal y el avance de internet, el consumo de televisión ha dejado de estar unido al televisor y ha llegado a los ordenadores, a los teléfonos móviles o a cualquier otro dispositivo con pantalla capaz de recibir señal audiovisual. Las cadenas de televisión han estado atentas a las nuevas posibilidades y han adecuado su estructura productiva a la emisión vía internet, implantando distintos tipos de software para garantizar la convergencia y aprovechar las posibilidades de operar con documentos y archi- 
vos digitales. En la primera fase del proceso digitalizaron los archivos para su ingesta, catalogación, almacenamiento y recuperación (DeBustos-Pérez-De-Salcedo, 2007) $\mathrm{y}$ en un estado posterior avanzaron en la modificación de las rutinas de producción para facilitar las tareas de publicación multiplataforma con las prestaciones que garantizan los CMS.

Las empresas de la Corporació Catalana de Mitjans Audiovisuals (CCMA) disponen de distintos programas para la elaboración y gestión de contenidos digitales: Digition se utiliza en Televisió de Catalunya (TVC), Dalet en Catalunya Ràdio y DeliverTy en la Corporació Catalana de Radio i Televisió Interactiva (Ccrtvi). A su vez, estos programas usan Digition como sistema de archivo con más de 60.000 horas de audiovisuales.

DeliverTy es el CMS utilizado para aprovechar el contenido elaborado originalmente para televisión en las diversas plataformas. Este software, que había sido comercializado en 2004 por la empresa Iris NewMedia, fue adquirido por la $C C M A$ para facilitar un trabajo independiente para cada medio de acuerdo con los elementos seleccionados por los administradores del gestor. Aunque en la actualidad DeliverTy ya no se comercializa, ha continuado desarrollándose $\mathrm{y}$ adecuándose a las necesidades de Televisió de Catalunya gracias al trabajo permanente del área técnica de Ccrtvi. La empresa que heredó el know-how de DeliverTy es Ctv (Communi.tv).

\section{http://communi.tv/}

Frente a otros productos del mercado, DeliverTy fue adquirido inicialmente porque permitía una adaptación progresiva, con lo cual a largo plazo economizaría tiempo y dinero a la organización. Además su flexibilidad facilitaba su adecuación a las necesidades de los profesionales de la información, y por tanto aumentaba las posibilidades de éxito en la primera fase de uso del software en las rutinas productivas de la cadena.

Con el paso del tiempo las nuevas versiones de Deliverty han conseguido un alto nivel de integración con los demás programas de gestión de contenido digital usados por la CCMA y con Digition. El CMS administra las piezas audiovisuales producidas por TVC y funciona como repositorio central de los archivos. Esta combinación facilita la gestión del material multimedia desde el CMS antes de su publicación en cualquier plataforma y con ello cubre todo el circuito de producción, desde la concepción de la pieza hasta su extracción y publicación.

Deliverty incorpora los metadatos ya existentes en el gestor documental que se encuentran relacionados con explotación del contenido, el formato, la duración, las restricciones geográficas de emisión o la proporción del aspecto (4:3 ó 16:9). Esta información facilita a los administradores la codificación, la decodificación y la distribución, pero en cambio, ofrece poca metainformación editorial de descripción del contenido, una carencia en la que trabaja el equipo técnico de Ccrtvi y que quedará superada a corto plazO.

\section{"Aunque Deliverty ha dejado de ofrecerse como un producto comercial, el área técnica de Ccrtvi ha continuado adecuándolo a las necesidades de TVC"}

Deliverty usa el programa Rhoz para transformar los archivos según su plataforma de salida. Los contenidos se almacenan en una base de datos que los retiene mientras llega el momento de su extracción. Esta base de datos es una réplica del repositorio central de la CCMA cuya función es proteger al sistema contra incidencias, agilizar la emisión y facilitar los cambios.

Los servicios de indexación y de búsqueda se controlan desde el software open source Lucen, que funciona con total autonomía sobre el gestor de contenidos y de esta manera evita que los cambios en los contenidos puedan afectar a la recuperación de los mismos. Las codificaciones se hacen con criterios de priorización establecidos previamente. De esta manera, por ejemplo, la emisión de noticias en internet siempre tiene prioridad sobre otros programas.

La infraestructura técnica que soporta las operaciones de Deliverty es compleja y costosa. De ahí que algunas de sus partes hayan sido adjudicadas por la CCMA vía concurso público a empresas externas. Por ejemplo la empresa Colt se encarga del hosting de Deliverty, y Atlas se ocupa del mantenimiento de los equipos. Este $C M S$ está preparado para que todas las piezas se emitan según el tipo de pantalla, teniendo en cuenta sus características particulares, como número de caracteres por línea, disposición de la retícula, tamaño o peso de la información global.

Las tareas de extracción son programadas con antelación mediante un planificador que ordena al sistema, por ejemplo, publicar un titular cada hora en un sitio web o en el teletexto de $T V C$. Aunque se trata de una acción mecánica, desde la Ccrtvi monitorizan el servicio de forma constante para que el contenido de audio y vídeo se integre correctamente en las plataformas preestablecidas.

Con la automatización de la emisión, Deliverty no sólo eleva la disponibilidad de la información, sino también el número de piezas multimedia en todas las webs de 
la $C C M A$ y hace posible la integración automática de vídeos en YouTube y otras plataformas de vídeo populares como Vimeo o DailyMotion. En el último rediseño del portal televisivo en febrero de 2008 este $C M S$ contribuyó a mejorar la organización de los contenidos y a extender los vídeos a casi todos los sitios web de programas. En el portal aumentan los servicios de participación como blogs, juegos, chats, foros y concursos, gestionados por los realizadores de los espacios televisivos, acreditados como administradores en Deliverty para editar, filtrar y emitir los contenidos generados por la audiencia.

Es importante resaltar que el software no participa en la administración de contenidos en redes sociales como Twitter o Facebook. Este tipo de servicios forman parte de un proyecto independiente elaborado por la Ccrtvi de gestión de comunidades online.

\section{Ciclo de vida de los contenidos online}

La producción de las cadenas de televisión se caracteriza por un alto grado de división del trabajo dentro de un circuito que combina los esfuerzos de productores, técnicos, realizadores, periodistas y profesionales de diversas áreas de la empresa. A este ecosistema productivo se suman las compañías externas que producen o coproducen para el medio. Para comprender la manera en que Deliverty interviene en este proceso adoptamos el modelo del ciclo de vida de los contenidos descrito por Hackos (1994, p. 26), según el cual cada pieza atraviesa un circuito de cinco fases: planificación, especificación, implementación, producción y evaluación.

Deliverty trabaja por medio de plantillas, donde los equipos de realización introducen las piezas para su emisión por internet, home media center y teléfonos móviles. Por ello antes de comenzar a usar el

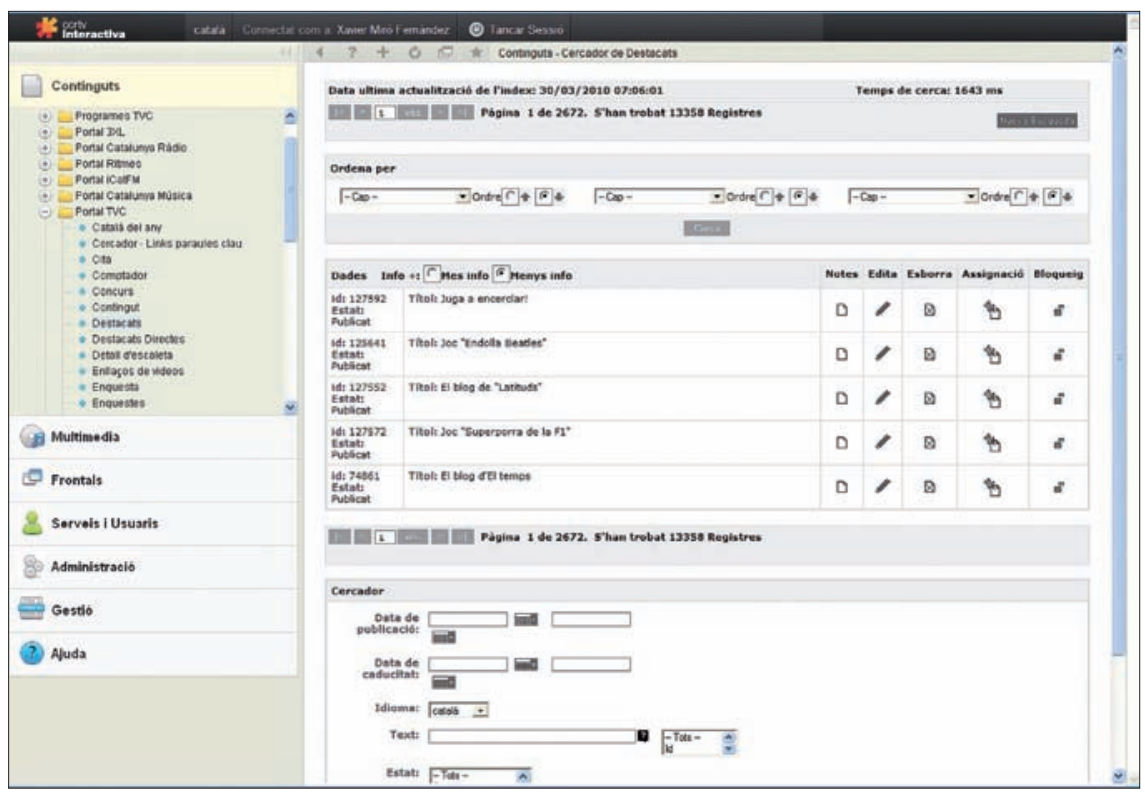

Figura 1. Interfaz de Deliverty. Imagen cedida por la Ccrtvi, 2010.

CMS se debe crear un dominio para cada programa dentro de Deliverty según las secciones, los contenidos y plataformas de emisión que ofrezca. Esta forma de trabajo hace posible la administración personalizada de la información dentro de un entorno de trabajo en red.

Para decidir los campos que tendrá la ficha de administración del contenido en Deliverty, los profesionales de la información deben conocer previamente las fortalezas de cada plataforma como sistemas de comunicación.

Los contenidos mutiplataforma deben ser acordados con la Ccrtvi, responsable de la implementación y la adaptación del sistema. En esta etapa los periodistas de TVC están abocados a entenderse y usar un lenguaje común con los responsables del área técnica de la Ccrtvi, una tarea compleja porque se trata de dos subculturas profesionales distintas. Para facilitar la comunicación dentro de estos grupos de trabajo multidisciplinares el diálogo se canaliza mediante interlocutores únicos nombrados como coordinadores del proyecto en representación de los colectivos durante el proceso de producción. Las ideas deben detallarse y expresarse de manera pormenorizada y por escri- to, para garantizar la correcta comprensión por parte de los diferentes integrantes de los equipos.

Una vez se ha decidido qué contenidos se emitirán y desde qué plataformas, las ideas se plasman en un documento interno que reúne las especificaciones del proyecto. En esta fase, profesionales de diversas áreas y perfiles como redactores, realizadores, técnicos, coordinadores de contenidos y personal de dirección, acuerdan las necesidades del proyecto y las posibilidades informáticas de su realización.

Este tipo de trabajo significa un cambio cultural notable en la organización, ya que como afirma Schein (1988) los profesionales forman sus métodos, valores y su propia imagen básica en torno a la tecnología que les es inherente y si la tecnología, como en este caso, sufre una transformación sustancial, los trabajadores de la empresa deben no sólo aprender nuevos métodos, sino que se necesita una redefinición de la propia institución para atender a las innovaciones y a los cambios culturales de fondo.

Durante el proceso de implementación se crean en Deliverty los espacios de trabajo para cada programa, se realizan ajustes y se 
proyectan las tareas de extracción automática del contenido. Después, los profesionales vinculados a la realización de los programas de televisión son dados de alta como administradores habilitados para clasificar y dirigir las piezas multimedia que producen. De esta manera pueden asignar, por ejemplo, a cada vídeo un titular, una entradilla, un texto y algunas imágenes asociadas, programar su hora y día de publicación online, los sitios web donde aparecerá, su ubicación en la página y el tiempo que será accesible.

Deliverty presenta distintos espacios de trabajo, según la finalidad con la que se use el sistema, pero es mediante el entorno denominado Frontals, con el que se ordenan los diferentes contenidos y se elige su lugar de publicación.

Para ordenar la presentación en el sitio web basta asignar un número que le indica al CMS el lugar de publicación. Así por ejemplo, el cero responde a una prioridad mínima que ordena al software insertar el contenido al final de la web y por el contrario, el 99 representa el máximo valor, con lo que el contenido se mostrará en la parte superior.

Aunque a simple vista la producción mediante Deliverty puede parecer sencilla, son frecuentes las preguntas de los realizadores de televisión que no están habituados a trabajar en un entorno online. Por ello, la Ccrtvi redacta manuales de usuario para cada programa que explican paso a paso la gestión de los contenidos digitales en este entorno. Así mismo, el equipo técnico ha mejorado la usabilidad del CMS aumentando los elementos gráficos para que la tarea de los administradores de la información sea más eficaz.

En la parte final del proceso encontramos la evaluación del sistema para identificar las incidencias técnicas e introducir o eliminar campos en función del progreso de cada proyecto. Con el fin de garantizar la correcta valoración del $C M S$, el área de arquitectura técnica de la Ccrtvi dispone de un grupo de profesionales encargados del seguimiento constante de todo el proceso, su rendimiento, usabilidad y funcionalidad.

\section{Conclusiones}

Desde el punto de vista estratégico, el uso de CMS puede ser considerado como una consecuencia lógica de la digitalización de los archivos. Una etapa donde la gestión documental ocupa un lugar destacado en la arquitectura de la producción. Como se ha visto en este trabajo, sin la existencia de Digition como repositorio central sería inviable la explotación de los contenidos en las nuevas plataformas. El fondo documental constituye una pieza estratégica de la producción televisiva, que ahora se prepara para afrontar la distribución multiplataforma.

En el seno de las organizaciones televisivas, la implementación de los CMS ha conseguido que la emisión online sea menos compleja. Este software abre nuevas posibilidades al sector audiovisual y facilita la implementación de contenidos en la Web y en otras plataformas digitales.

\section{"Técnicos y periodistas están abocados a entenderse y usar un lenguaje común"}

La adopción del sistema Deliverty en TVC ha reducido los costes de la producción destinada a internet y ha rentabilizado los recursos humanos y técnicos de la empresa. En referencia a las rutinas productivas, la utilización del CMS ha comportado cambios notables al permitir a los profesionales trabajar en red, controlar el ciclo de vida de los productos y automatizar la emisión.

La implementación de Deliverty en $T V C$ ha superado ya los obstáculos técnicos y el esfuerzo se centra ahora en la adaptación de la interfaz del sistema para hacerla más intuitiva y amigable a los usuarios y en afianzar el proceso de convergencia. Una convergencia que implica avanzar en una nueva cultura organizacional que permita aprovechar las fortalezas y las sinergias de los distintos equipos humanos, con el objetivo de producir contenidos audiovisuales de calidad para su distribución en las diversas plataformas disponibles.

\section{Notas}

1. Una parte de los resultados presentados procede del proyecto Entorno cross media: transformaciones organizativas y productivas en los grupos radiotelevisivos (CSO2009-09367), financiado por el Ministerio de Ciencia e Innovación dentro del Plan Nacional I+D+i 2009-2011.

2. Inicialmente Deliverty fue comercializado bajo el nombre DeliverTy, pero en la actualidad dentro de $T V C$ se escribe sin la $T$ mayúscula.

\section{Bibliografía}

Benzing, Matt. "Luwak: a content management solution". Library hi tech, 2006, v. 24, n. 1, pp. 8-13.

De-Bustos-Pérez-De-Salcedo, Pilar. "Sistemas integrados y gestión documental. La experiencia en Telecinco". El profesional de la información, 2007, sept.-oct., v. 16, n. 5, pp. 450-455.

http://www.elprofesionaldelainformacion.com/ contenidos/2007/septiembre/07.pdf

Hackos, JoAnn T. Managing your documentation projects. New York: Wiley, 1994. ISBN 0471-59099-1.

Jeffery-Poulter, Stephen. "Creating and producing digital content across multiple platforms". Journal of media practice, 2003, v. 3, n. 3, pp. 155-164.

Manovich, Lev. Software takes command, 2008.

http://lab.softwarestudies.com/2008/11/soft book.html

Schneider, Stephan. "Requirements and approaches for a content management service". En: Intl. conf. on automated solutions for cross media content and multi-channel distribution, 2008. pp. 127-132.

Seadle, Michael. "Content management system". Library hi tech, 2006, v. 24, n. 1, pp. 5-7.

Schein, Edgar H. La cultura empresarial y el 
liderazgo: una visión dinámica. Plaza \& Janes Editores: Esplugues de Ll., 1988, ISBN 84-0136107-9.

Serrano-Cobos, Jorge. "Evolución de los sistemas de gestión de contenidos $(C M S)$. Del mainframe al open source". El profesional de la información, 2007, mayo-junio, v. 16, n. 3, pp. 213-215.

http://www.elprofesionaldelainformacion.com/ contenidos/2007/mayo/05.pdf
Vila, Pere. "Convergència de continguts". Quaderns del CAC, Convergència tecnològica i audiovisual, 2009, jul. 2008-jun. 2009, v. 31-32, pp. 71-76.

http://dialnet.unirioja.es/servlet/articulo?codigo $=3048626 \&$ orden $=222954 \&$ info $=$ link

Rosa Franquet, María-Isabel Vi-
lla-Montoya. Dept. de Comunicació Audiovisual i de Publicitat, Univ. Autònoma de Barcelona. Edif. 1, Fac. de Ciències de la Comunicació, Campus de Bellaterra, 08193 Bellaterra (Barcelona). rosa.franquet@uab.cat mariaisabel.villa@uab.cat

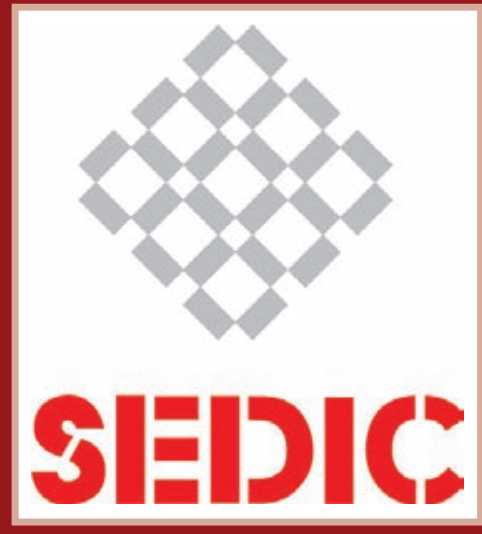

\section{PREMIO DE ENSAYO $S=$ DIC "TERESA ANDRÉg" 2010}

Resumen de las bases

- Pueden participar personas de cualquier nacionalidad.

- Los trabajos deben ser inéditos, en castellano, y tener entre 50.000 y 100.000 palabras.

- Tema: Reflexión con perspectiva sobre cualquier asunto relacionado con bibliotecas, archivos y otros servicios de información, los profesionales que los atienden y su papel en la sociedad pasada, presente y futura.

- Plazo límite: 15 de diciembre de 2010.

- Premio: 3.000 euros y publicación del ensayo como libro en Ediciones Trea.

Bases completas

http://www.sedic.es/premio-ensayo-SEDIC-teresa-andres-convocatoria-2010.asp

Teresa Andrés Zamora (1907-1946) fue una profesional ejemplar. En 1931 ingresó en el Cuerpo Facultativo de Archiveros, Bibliotecarios y Arqueólogos (obtuvo el primer puesto de las oposiciones) y fue destinada a la Biblioteca del Palacio Nacional. Dominaba francés, inglés y alemán. En 1932 viajó a Alemania donde trabajó en varias bibliotecas y museos. Durante la guerra fue responsable de la sección de bibliotecas de Cultura Popular, creando bibliotecas en hospitales, batallones y hogares del soldado.

\section{Comité organizador}

Paz Fernández y Fernández-Cuesta Directora del Servicio de Bibliotecas de la Fundación Juan March. Vocal de la Junta directiva de SEDIC

\section{María Jesús del Olmo}

Directora Centro de Recursos Informativos de la Embajada de EE.UU. Secretaria general de la Junta directiva de SEDIC

\section{Más información}

\section{Rosa Martínez}

